\title{
Microscopy to Support Trace Screening of Contraband, Including Explosives and Illicit Drugs
}

\author{
Jennifer R. Verkouteren ${ }^{1}$ and Edward Sisco ${ }^{1}$ \\ 1. Surface and Trace Chemical Analysis Group, National Institute of Standards and Technology, \\ Gaithersburg, MD USA.
}

Screening technologies deployed for detecting contraband, including explosives and illicit drugs, rely predominantly on the physical collection of trace particle residues. The bulk materials typically contain crystalline particles of the target contraband in a complex matrix, and handling, e.g. bomb making, can lead to formation of trace residues by fingerprint transfer from contaminated hands. Fundamental to determining the likelihood of detection in a screening application is understanding the physical and chemical characteristics of these residues; how many contraband particles are expected, what is their size, and what is the environmental background expected. Our earlier work in this area dealt with optical and SEM measurements of the explosives 1, 3, 5-trinitroperhydro-1, 3, 5-triazine (RDX) and pentaerythritol tetranitrate (PETN). We developed an optical microscopy approach that used the birefringence of the explosives particles to image and size the particles over the area of a fingerprint within a matrix of fingerprint oils and the plastic components of the explosives formulation [1,2]. We typically limit the minimum particle size detected by optical microscopy to 4 micrometers based on magnification and image resolution parameters. Although smaller particles exist, as determined by SEM analysis, they do not contribute significantly to the mass of the particle deposit for the fingerprints we have examined. This is because explosives formulations, particularly military grade, typically contain large particles (diameters $>100 \mu \mathrm{m}$ ) and any large particles in the fingerprints will dominate the mass distribution.

Our current efforts involve mapping the positions and sizes of explosives particles within a fingerprint to serve as standards for measuring sampling efficiency (Figure 1a). Particle size can have a large effect on sampling efficiency, particularly for aerodynamic removal and collection, and mapping the deposit before and after sampling provides collection efficiency with respect to particle size. Mass-based collection efficiency can also be determined from the microscopic measurements by calculating the mass for each particle using a fixed factor for particle height with respect to measured diameter. This approach is shown in Figure 1b, where the combined mass of PETN particles in a sequence of 10 fingerprints (made after contaminating the finger one time) was determined from the microscopic measurements, and then by chemical extraction and analysis. The comparison shows that the microscopic approach has errors ranging from $4 \%$ to $50 \%$ with respect to the chemical extraction data, which is sufficient for collection efficiency measurements. The microscopic approach allows for nondestructive determination of mass (and particle size and distribution) for preparation of standard samples, and allows for the use of the real threat materials rather than simulants. We are evaluating ways of standardizing the fingerprint preparations using artificial fingers, although the mass deposited in a sequence is still quite variable, as seen in Figure 1b. A benefit of using artificial fingers is that the total amount of material loaded onto the finger can be determined, and the transfer efficiency per print can be calculated. This provides a mechanism to predict the mass (within an order of magnitude) expected in an extended sequence of fingerprints made after a single contamination.

For the contraband described above, the explosive compound itself is used for detection in screening 
activities and is present as discrete particles. In the case of gunpowders, used frequently in pipe bombs, there are a range of chemical compounds indicative of the formulations that might be used in a detection scheme. The particles that are shed from the larger grains of black powders and black powder substitutes may not be homogeneous, and mapping these particles requires an additional component of chemical discrimination. We are using microXRF and SEM/EDS analysis for this purpose and will show the differences for traditional black powders compared with the substitutes, with the substitutes exhibiting much more heterogeneity than black powders. Finally, there are types of contraband that may produce residues by means other than fingerprint transfer, such as by aerosol transfer of fine, loose powders. We have demonstrated a trace screening approach to detecting fentanyl and fentanyl analogues in street samples, which are present as powders mixed with significant ratios of cutting agents [3]. Sampling residue off the bag to identify the contents removes the hazard of exposure from opening the bag, but requires an understanding of how much residue is commonly expected. The material on the outside of bags is likely to be present from both fingerprint transfer and from aerosol deposition of the fine powders. We will describe preliminary results to evaluate locations and amounts of trace contamination on the outside of drug exhibits.

\section{References:}

[1] J R Verkouteren, Journal of Forensic Sciences 52 (2007), p. 335.

[2] J R Verkouteren, J L Coleman, I Cho, Journal of Forensic Sciences 55 (2010), p. 33.

[3] E Sisco, J R Verkouteren, J Staymates, J Lawrence, Forensic Chemistry 4 (2017), p. 108.
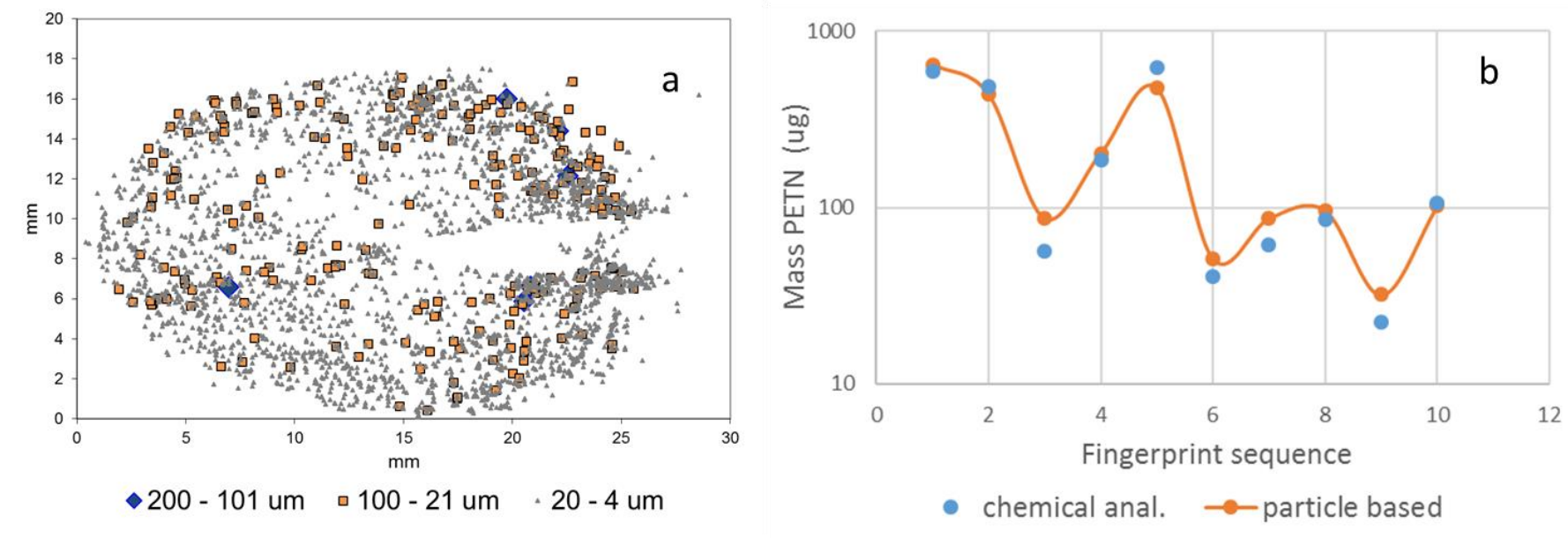

Figure 1. (a) Locations and binned sizes of RDX particles in a fingerprint produced after handling the explosive formulation C-4. (b) Comparison of masses of PETN in fingerprints made in sequence calculated from particle data and directly measured by chemical extraction and analysis. 